\title{
Phenotypic Differences Between Natural and Induced Variants of Botrytis cinerea
}

\author{
By MORRIS GRINDLE \\ Department of Genetics, The University, Sheffield S10 2TN
}

(Received 16 August 1978)

\begin{abstract}
Wild isolates of Botrytis cinerea were analysed to assess their suitability for genetic studies. When compared on synthetic and artificial media under laboratory conditions, the isolates differed in rate of growth, conidiation, sclerotia production and resistance to benomyl. Asexual progeny of individual cultures were sometimes phenotypically diverse, indicating that they could be heterokaryotic or heteroplasmic. Attempts to derive auxotrophic, nystatin-resistant and benomyl-resistant mutants from mutagen-treated conidia were not successful: only morphological and spore colour variants were recovered, and most of the morphological variants were phenotypically unstable. Cultures produced both macroconidiophores and microconidiophores. Macroconidia were about $10 \mu \mathrm{m}$ long $\times 8 \mu \mathrm{m}$ wide, contained 1 to 12 nuclei (mean $3 \cdot 4$ ) and usually germinated readily on agar media. Microconidia were about $3 \mu \mathrm{m}$ diameter, uninucleate and rarely germinated on agar media. The perfect state, Botryotinia fuckeliana, was not recovered from mixed inocula of the wild strains. The significance of the results is discussed in relation to the use of $B$. cinerea for laboratory studies and the possible genetic basis of natural variations.
\end{abstract}

\section{INTRODUCTION}

Few fungal plant pathogens have been subjected to comprehensive genetic analysis (for example, see Day, 1974; Webster, 1974; Burnett, 1975). Fundamental genetic questions relating to most pathogenic species remain unanswered. For instance, what is the genetic basis for phenotypic variations among natural populations? What are the genetic factors controlling outcrossing, and how much genetic variation can be released by sexual or parasexual recombination? Are wild strains haploid or diploid, homokaryotic or heterokaryotic, homothallic or heterothallic? In the absence of genetic data, taxonomic divisions are often based solely on morphological criteria and host specificity, and simple genetic variants of interbreeding populations might be classified as distinct species.

The aim of this investigation was to assess the suitability of a common pathogenic filamentous fungus, Botrytis cinerea ('grey mould'), for genetic studies. Botrytis cinerea was chosen for four main reasons: it is a facultative pathogen that can be cultured on artificial media in the laboratory; wild isolates are abundant on a wide variety of host plants; a perfect state has been recorded (Botryotinia fuckeliana, an operculate discomycete; see Hennebert, 1973) which, even though it is rare, means that it is feasible to derive sexual progeny; it is economically important, causing losses to fruit and vegetable crops.

The relationship between $B$. cinerea and Botryotinia fuckeliana has been demonstrated by Groves \& Drayton (1939), Groves \& Loveland (1953) and Polach \& Abawi (1975). The common conidial state, $B$. cinerea, consists of coenocytic mycelia that give rise to asexual spores and sclerotia (tough, black, irregular-shaped aggregations of hyphae). Sclerotia can withstand harsh environmental conditions, and the fungus probably overwinters in this 
Table 1. Strains of Botrytis cinerea

\begin{tabular}{|c|c|c|}
\hline Strain & Isolation no. & Source* \\
\hline 282 & 160.282 & Aesculus hippocastanum, Kew, 1971 \\
\hline 465 & 100.465 & Punica granatum fruit, India, 1963 \\
\hline 558 & 169.558 & Lycopersicon esculentum, Perthshire, 1972 \\
\hline 649 & 142.649 & Vicia faba, Berkshire, 1958 \\
\hline 1 & WA3 & Onion, Wellesbourne, Warwick, 1977 \\
\hline 2 & WA6 & Onion, Wellesbourne, Warwick, 1977 \\
\hline 3 & WA8 & Onion, Wellesbourne, Warwick, 1977 \\
\hline 4 & WB2 & Onion, Wellesbourne, Warwick, 1977 \\
\hline 5 & WB4 & Onion, Wellesbourne, Warwick, 1977 \\
\hline 9 & WC3 & Onion, Wellesbourne, Warwick, 1977 \\
\hline 10 & $\mathrm{GC}$ & Greenhouse cucumber, Sheffield, 1977 \\
\hline 11 & GT1 & Greenhouse tomato, Sheffield, 1977 \\
\hline 12 & HB2 & Broad bean, Huddersfield, 1977 \\
\hline 14 & SB2 & Broad bean, Sheffield, 1977 \\
\hline
\end{tabular}

* Strains 282, 465, 558 and 649 were supplied by the Commonwealth Mycological Institute, Kew, Surrey. Strains WA, WB and WC were from three different fields of onion at the National Vegetable Research Station.

form to provide fresh colonies and spores in spring. The larger and more obvious spores (macroconidia) are easily disseminated to infect new host plants. The smaller spores (microconidia) probably function as spermatia in sexual reproduction (Drayton, 1932). The perfect state can be derived from sclerotia by applying conidia to their surface: cultures of $B$. cinerea are not self-fertile, indicating that it is a heterothallic organism, but the number of mating type loci and alleles is not known. Fertilized sclerotia occasionally give rise to stalked apothecia containing asci. The lengthy and involved procedure for producing apothecia under laboratory conditions (Groves \& Loveland, 1953) could reflect an innate property of the organism or simply insufficient data on its requirements for sexual reproduction.

The inadequacy of our genetic knowledge of $B$. cinerea is exemplified by Webster's (1970) statement: 'It is probable that the name Botrytis cinerea is a collective name used to describe a number of closely similar, but genetically distinct, species, possibly with distinct apothecial states'. It is hoped that the information in this report will provide a foundation on which detailed genetic studies can be based and statements like the one above can be clarified.

\section{METHODS}

Strains. Cultures were obtained from the Commonwealth Mycological Institute, Kew, Surrey, and were isolated from diseased plant material. Their designations and sources are given in Table 1 . The strains used for comparative studies were single conidial isolates of the original cultures, maintained on agar media at $5^{\circ} \mathrm{C}$ or 20 to $25^{\circ} \mathrm{C}$.

Media. Stocks were transferred regularly to dishes or slants of synthetic media (MM and CM) and artificial medium (PDA). The minimal medium (MM; Vogel, 1964) contained $1.5 \%(\mathrm{w} / \mathrm{v})$ sucrose as the carbon source, and the complete medium (CM) consisted of MM supplemented with $0.5 \%(\mathrm{w} / \mathrm{v})$ yeast extract and casein hydrolysate. PDA contained $4 \%(\mathrm{w} / \mathrm{v})$ potato extract $($ Difco) and $1.5 \%(\mathrm{w} / \mathrm{v})$ glucose, and was adjusted to $\mathrm{pH} 6.0$ with $10 \mathrm{M}-\mathrm{NaOH}$.

Strains were compared for growth and morphology on MM, MM supplemented with benomyl (supplied as the garden fungicide Benlate by Pan Brittanica Industries, Hertfordshire), PDA, Neurospora medium SC (Westergaard \& Mitchell, 1947), Aspergillus media (Pontecorvo et al., 1953), synthetic media X and Y (Last \& Hamley, 1956), V8 agar (Peacock, 1966) and malt extract agar (Oxoid). Media were solidified with $2 \%$ (w/v) agar.

Slow-growing, compact colonies were obtained on MS and CMS media, which comprised MM and CM, respectively, supplemented with $1 \%(\mathrm{w} / \mathrm{v})$ sorbose and $0 \cdot 1$ to $0 \cdot 2 \%(\mathrm{w} / \mathrm{v})$ sucrose as carbon source; sorbose has a toxic effect on Neurospora crassa (Davis \& de Serres, 1970). Compact colonies were obtained also on MM or CM supplemented with G3300 (alkyl aryl sulphonate; Honeywill Atlas, Surrey) at 0.25 g 1-1; G3300 causes reduced growth of Aspergillus nidulans (Majerfeld \& Roper, 1978). 
Mutagenesis. Conidia from young cultures on MM or FDA were suspended in $1 \%(\mathrm{w} / \mathrm{v})$ saline and exposed to ultraviolet (u.v.) radiation, ethyl methanesulphonate or the acridine mustard ICR-170 as described by Grindle (1974). Samples were removed at intervals, spread on dishes of CMS and incubated at $25{ }^{\circ} \mathrm{C}$ to determine survival curves. Additional samples, removed when about $50 \%$ and $10 \%$ of the conidia were viable, were used to select mutants.

To isolate morphological variants, conidia were incubated on dishes of CMS, and hyphae from the resultant colonies were transferred to slants of $\mathrm{CM}$. Hyphae and conidia from the cultures in $\mathrm{CM}$ slants were inoculated on to dishes of $\mathrm{MM}$ and $\mathrm{CM}$ and incubated at 20 to $25^{\circ} \mathrm{C}$ to obtain colonies that could be compared easily.

To select resistance mutants, conidia were suspended in cool $\left(45^{\circ} \mathrm{C}\right) \mathrm{CMS}$, poured into dishes and incubated for 24 to $36 \mathrm{~h}$ at $22{ }^{\circ} \mathrm{C}$. The dishes of germinated conidia were overlayered with cool CMS supplemented with 3,6 and $10 \mu \mathrm{g}$ benomyl ml ${ }^{-1}$ or cool CMS supplemented with 5 and 10 units nystatin ml-1. Nystatin is a polyene antibiotic (supplied as mycostatin by E. R. Squibb \& Sons, Speke, Liverpool). The overlayered dishes were incubated at $22{ }^{\circ} \mathrm{C}$, and colonies that grew through the benomyl or nystatin layer were transferred to slants of $\mathrm{CM}$ to provide material for further analysis.

Attempts were made to select auxotrophic mutants using the method given above for isolating morphological variants, and also the technique of 'filtration enrichment'. Mutagen-treated conidia were incubated in flasks of liquid MM on a mechanical shaker at $26^{\circ} \mathrm{C}$; at $24 \mathrm{~h}$ intervals, the medium was filtered through glass wool to remove germinated conidia. After three or four filtrations, the MM was supplemented with sorbose, yeast extract and casein hydrolysate to permit limited growth of the remaining conidia. Colonies in the medium were removed with a Pasteur pipette and transferred to slants of CM to produce cultures for further growth tests.

Selection experiments. Mass-hyphal and mass-conidial inocula of wild strains were incubated on dishes of MM, CM and CM supplemented with $p$-fluorophenylalanine (PFA; Koch-Light) at 40 to $160 \mu \mathrm{g} \mathrm{ml}^{-1}$. Colonies that developed after incubation at 20 to $25^{\circ} \mathrm{C}$ were scrutinized for patches or sectors with unusual morphology. Tiny samples of conidia or hyphae were removed from the colonies with fine glass needles and incubated on dishes of MM, CM, X or Y media.

Suspensions of conidia from cultures of wild strains on MM and PDA were spread on dishes of CMS. Individual germinated conidia were transferred to dishes of $\mathrm{MM}$ and $\mathrm{CM}$ and incubated at 20 to $25^{\circ} \mathrm{C}$.

The colonies derived from conidial and hyphal inocula were compared with those of the parental wild strain to see whether they differed in growth rate or morphology.

Analysis of conidia. Spore suspensions consisting mainly of macroconidia were obtained from vigorous cultures on slants or dishes of MM and PDA. Suspensions with a high microconidial content were obtained from dishes of water agar and from flasks of dilute liquid MM $(10 \%$ of the usual concentration of salts and sucrose) inoculated with macroconidia. After 14 to $30 \mathrm{~d}$ incubation at $22{ }^{\circ} \mathrm{C}$, conidia on the dishes of media were washed off with $1 \%(\mathrm{w} / \mathrm{v})$ saline, and conidia in the flasks were filtered through glass wool.

Microconidia were separated from the macroconidia and hyphal fragments in crude spore suspensions by filtration or centrifugation. They were trapped on Millipore filters of $0.45 \mu \mathrm{m}$ pore size after passage through filters of $8 \mu \mathrm{m}$ pore, or they were sampled from sucrose gradients in $5 \mathrm{ml}$ centrifuge tubes. For centrifugation, the spore suspensions were layered on 10 to $40 \%$ (w/v) sucrose gradients, accelerated to $1100 \mathrm{~g}$ and immediately run down without braking; fractions were removed with a hypodermic needle attached to a peristaltic pump (Watson-Marlow, Falmouth, Cornwall). The centrifugation system proved to be the more effective.

Conidia and conidiophores were examined with a Kyowa SMKE microscope fitted with an eyepiece micrometer and a Nikon EFM photomicrographic camera. Dry conidia were stained on cover slips with Azure A using the method of Huebschman (1952) or by the Feulgen reaction, with Carnoys fixative, as outlined by Greenwood \& Berlyn (1968). Macroconidia were germinated on slides of water agar incubated at $22{ }^{\circ} \mathrm{C}$ in Petri dishes containing deionized water and inorganic salts (Weast, 1967) to maintain relative humidities of 70 to $100 \%$; they were stained with cotton blue.

Crossing experiments. Individual strains and mixtures of strains were cultured at 15 to $25^{\circ} \mathrm{C}$ on dishes of MM, CM and PDA and in flasks of sterile garden soil. Additionally, colonies were incubated at $22^{\circ} \mathrm{C}$ on dishes of MM and PDA for 7, 14 and $28 \mathrm{~d}$, and suspensions of microconidia and macroconidia from different wild isolates were applied to the sclerotia. 
Table 2. Rate of growth, density of conidia, number of sclerotia, and size of macroconidia of wild strains of Botrytis cinerea on agar media

All colonies were incubated on Petri dishes of $85 \mathrm{~mm}$ diameter at $22^{\circ} \mathrm{C}$; growth measurements were commenced at day 2; conidia and sclerotia determinations were done at day 15.

\begin{tabular}{|c|c|c|c|c|c|}
\hline \multirow[b]{2}{*}{ Strain } & \multicolumn{4}{|c|}{ Synthetic minimal medium (MM) } & \multirow{2}{*}{$\begin{array}{c}\mathrm{MM+} \\
\text { benomyl } \\
\text { Rate of } \\
\text { growth* } \\
\left(\mathrm{mm} \mathrm{d}^{-1}\right)\end{array}$} \\
\hline & $\begin{array}{l}\text { Rate of } \\
\text { growth* } \\
\left(\mathrm{mm} \mathrm{d}^{-1}\right)\end{array}$ & $\begin{array}{l}\text { Density of } \\
\text { conidia } †\end{array}$ & $\begin{array}{l}\text { Number of } \\
\text { sclerotiat }\end{array}$ & $\begin{array}{l}\text { Size of macroconidia } \S \\
(\mu \mathrm{m})\end{array}$ & \\
\hline 558 & 23 & $* * * *$ & $*$ & $9.4(1.1) \times 7.7(0.6)$ & 14 \\
\hline 649 & 13 & $* * * *$ & 0 & $9.0(1.0) \times 7.3(0.6)$ & 14 \\
\hline 1 & 25 & $* *$ & $* *$ & $9.0(1.0) \times 7.7(0.7)$ & 15 \\
\hline 2 & 22 & $* *$ & $* * * *$ & $10.0(1.4) \times 7.9(0.8)$ & 10 \\
\hline 3 & 21 & $*$ & $* * * *$ & $9.6(1.3) \times 8.1(0.8)$ & 9 \\
\hline 4 & 18 & $* * *$ & 0 & $8.8(1.0) \times 7.0(0.4)$ & 17 \\
\hline 5 & 23 & $* * *$ & $* * *$ & $10.6(1.4) \times 8.5(0.7)$ & 13 \\
\hline 9 & 20 & $*$ & $* *$ & $9.3(1.2) \times 7.8(0.9)$ & 22 \\
\hline 10 & 24 & $* * *$ & $* *$ & $9.9(0.9) \times 8.1(0.7)$ & 26 \\
\hline 11 & 21 & $* *$ & $* *$ & $10.1(1.6) \times 7.9(0.9)$ & 22 \\
\hline 12 & 23 & $*$ & $* *$ & $10 \cdot 2(1 \cdot 1) \times 8.4(0 \cdot 7)$ & 19 \\
\hline 14 & 17 & $* *$ & $* *$ & $9.0(1 \cdot 1) \times 7.5(0.8)$ & 12 \\
\hline
\end{tabular}

\begin{tabular}{|c|c|c|c|c|}
\hline \multirow[b]{2}{*}{ Strain } & \multicolumn{4}{|c|}{ Potato glucose agar (PDA) } \\
\hline & $\begin{array}{l}\text { Rate of } \\
\text { growth* } \\
\left(\mathrm{mm} \mathrm{d}^{-1}\right)\end{array}$ & $\begin{array}{c}\text { Density of } \\
\text { conidia } †\end{array}$ & $\begin{array}{c}\text { Number of } \\
\text { sclerotia }\end{array}$ & $\begin{array}{l}\text { Size of macroconidia } \S \\
(\mu \mathrm{m})\end{array}$ \\
\hline 558 & 27 & $* * * *$ & $*$ & $9.4(1.3) \times 7.5(0.6)$ \\
\hline 649 & 16 & $* * * *$ & 0 & $9.4(1.3) \times 7.4(0.7)$ \\
\hline 1 & 33 & $* *$ & $* *$ & $9.6(1.0) \times 7.8(0.7)$ \\
\hline 2 & 33 & $*$ & $* * * *$ & $10.2(1.4) \times 8.4(0.8)$ \\
\hline 3 & 34 & $* *$ & $* * * *$ & $10.8(1.1) \times 9.1(1.0)$ \\
\hline 4 & 31 & $* * * *$ & $* * *$ & $8.1(1.0) \times 6.9(0.5)$ \\
\hline 5 & 30 & $* * * *$ & $* *$ & $10.7(1.0) \times 8.6(0.8)$ \\
\hline 9 & 31 & $*$ & $* *$ & $9.9(1.2) \times 8.3(0.6)$ \\
\hline 10 & 29 & $* * * *$ & $*$ & $10.4(1.0) \times 7.9(0.7)$ \\
\hline 11 & 29 & $* * *$ & ** & $10.8(1.4) \times 8.0(1.0)$ \\
\hline 12 & 23 & $* *$ & $* *$ & $9.9(0.8) \times 8.3(0.7)$ \\
\hline 14 & 30 & $* * * *$ & ** & $9.7(1.3) \times 8.0(0.8)$ \\
\hline
\end{tabular}

* Increase in colony diameter; mean of three replicates.

$\dagger$ Amount of colony covered by conidiophores (sparse* to complete****); mean of three replicates.

+ Number of sclerotia per colony $\left(5-10^{*}\right.$ to $\left.120-150^{* * * *}\right)$; mean of three replicates.

$\S$ Length $\times$ width; means (and standard deviations) of 40 conidia. The overall means are $9.7 \times 7.9 \mu \mathrm{m}$.

|| Benomyl [methyl(1-butylcarbamoyl)benzimidazol-2-ylcarbamate] at $0 \cdot 1 \mu \mathrm{g} \mathrm{ml}^{-1}$. Colonies on benomylsupplemented MM were morphologically identical to those on MM.

\section{RESULTS AND DISCUSSION}

\section{Growth conditions}

Strains $282,465,558$ and 649 were tested for growth on various media at 15 to $37^{\circ} \mathrm{C}$ to establish the most convenient regime for manipulating strains in the laboratory. They grew poorly at the higher temperatures, on malt extract and V8 agar, and with glycerol or starch as carbon source. Vigorous cultures were obtained at 15 to $30^{\circ} \mathrm{C}$, on $\mathrm{MM}, \mathrm{CM}, \mathrm{SC}, \mathrm{X}, \mathrm{Y}$, Aspergillus media and PDA, and with sucrose or glucose as carbon source. As a result of these tests, MM and PDA, and temperatures of 20 to $25^{\circ} \mathrm{C}$, were chosen for routine laboratory studies of $B$. cinerea.

The synthetic medium MM with sucrose as carbon source was chosen for five reasons: it is a defined medium prepared from simple materials that can be stored as a concentrated 



Figs 1 to 6. Morphological differences between wild strains of $B$. cinerea on MM (top row) and PDA (bottom row). All colonies were derived from mass-hyphal inocula, incubated at $22^{\circ} \mathrm{C}$ for 30 d. Petri dishes are $85 \mathrm{~mm}$ diameter. Strain 2 (Figs 1, 4); strain 11 (Figs 2, 5), the greater conidiation and size of sclerotia on PDA is typical of most wild strains; strain 14 (Figs 3,6).

solution; it supports good growth, conidiation and sclerotia production; it can be supplemented with specific chemicals for investigating nutritional mutants; it enables the detection of minor phenotypic differences between strains; its properties as a growth substrate are well documented (Davis \& de Serres, 1970). PDA was the best medium for rapid production of vigorous cultures with abundant conidia.

Media that promote rapid growth and conidiation are often not suitable for the isolation and analysis of genetic variants and sexual progeny. There is a need for media which limit growth substantially so that numerous isolates can be cultured on a Petri dish. Most of the wild strains formed neat, compact colonies on the sorbose media MS and CMS; the size of the colonies could be manipulated by varying the proportions of sorbose to sucrose. Strains that produced small diffuse colonies with irregular outlines on the sorbose media were easier to analyse on MM or CM supplemented with G3300.

\section{Phenotypic differences between wild strains}

Hyphal inocula, about $8 \mathrm{~mm}^{3}$, were transferred from the frontier of young cultures on PDA to the centre of dishes of agar media and incubated at $22{ }^{\circ} \mathrm{C}$. The resultant colonies were analysed for differences in rate of growth, conidia and sclerotia development, and size of macroconidia (Table 2). There were substantial strain differences in morphology (Figs 1 to 6), growth rate, density of conidiophores and sclerotia, and resistance to benomyl, but only minor differences in macroconidial size (Table 2). Differences between strains were usually more pronounced on MM than on PDA, particularly with respect to their rates of growth and pigmentation (e.g. strain 649 produced a diagnostic red pigment on MM, but not on PDA). PDA was superior to MM for deriving vigorous cultures: growth was faster, 
conidia were produced sooner and in greater numbers, and the sclerotia were larger (Figs 1 to 6). These phenotypic differences between strains were reproducible so long as large mass-hyphal inocula were used to derive colonies for the measurements; colonies derived from individual conidia or a few hyphae sometimes varied substantially (see later).

Three of the isolates were unaffected by the systemic fungicide, benomyl; strains 9,10 and 11 continued to grow normally on $\mathrm{MM}$ containing $500 \mu \mathrm{g}$ benomyl ml-1. The concentrations of benomyl needed to prevent growth of the other isolates were $0.2 \mu \mathrm{g} \mathrm{ml}^{-1}$ (strains $558,1,2,3,5$ and 14) and $0 \cdot 3 \mu \mathrm{g} \mathrm{ml}^{-1}$ (strains 649, 4 and 12). Benomyl-resistant strains of various fungi have been obtained from field populations (see Dekker, 1977). The presence of three resistant strains in such a small sample of natural isolates of $B$. cinerea [and of one resistant strain among the three isolates of Botrytis squamosa that were recovered from the onion crop at Wellesbourne in 1977 (unpublished data)] suggests that extensive use of benomyl is applying heavy selection pressure in favour of spontaneous mutants. There is also the possibility that benomyl is inducing gene mutations (Dassenoy \& Meyer, 1973) or chromosomal instability (Hastie, 1970).

Since the wild strains differ for most of the phenotypic characters, including quantitative traits under polygenic control, there must be considerable genetic diversity among wild populations of $B$. cinerea. Such diversity can be generated by mutation and by gene recombination between variants. But what are the relative contributions of these factors to genetic diversity in $B$. cinerea populations? Can fungicide resistance, for example, result from mutations in more than one gene, and can these genes be recombined via a sexual or parasexual cycle to produce strains with different levels of resistance? It was felt that the mutation versus recombination question would be answered most effectively by analysing laboratory mutants rather than natural variants. Accordingly, attempts were made to induce mutants carrying suitable genetic markers for monitoring gene flow and recombination between strains.

\section{Induction of mutants}

The conidia of $B$. cinerea wild strains were sensitive to all three mutagens, and unremarkable dose-effect curves were obtained as a prelude to mutant selection procedures. With u.v.-irradiation, for instance, the $L_{10}, L_{50}$ and $L D_{90}$ times were approximately 20 , 120 and $360 \mathrm{~s}$, respectively. Despite the lethal effects of the mutagens, very few useful mutants were selected from the surviving progeny.

In the search for morphological mutants among progeny on dishes of CMS, many variants of strains 558 and 649 were recovered that produced abnormal colonies on CM. The variants differed from the parental strains in colonial morphology, rate of growth, conidiation and pigmentation. On repeated subculture by mass-hyphal transfers, however, most of the variants proved to be phenotypically unstable and they soon resembled the original wild strain.

No resistance mutants of strains 649,5 and 10 were recovered from a total of almost $3 \times 10^{7}$ viable, mutagen-treated conidia that were incubated in nystatin-supplemented media. Using the same mutagenic and selection methods with conidia of Neurospora crassa, nystatin-resistant mutants have been recovered with high frequencies (Grindle, 1973, 1974). Nystatin kills normal strains of $N$. crassa by binding to the ergosterol component of cell membranes so that the membrane is distorted and cell contents leak out. Resistant mutants carry recessive gene mutations causing blocks in ergosterol biosynthesis (Grindle \& Farrow, 1978). Cell extracts of $B$. cinerea cultures, analysed as described by Grindle (1973), were found to contain ergosterol: it seems unlikely, therefore, that nystatin-resistant mutants have not been recovered because $B$. cinerea lacks appropriate mutable genes. It also seems unlikely that no mutants were obtained because multinucleate macroconidia were used for the mutagenic studies: the mutants of $N$. crassa were derived from macroconidia containing similar numbers of nuclei (the mean number of nuclei in stained macroconidia of $B$. cinerea was $3 \cdot 4)$. 
Morphological variants were isolated from nystatin media that had lost its antibiotic properties after 4 weeks incubation. Most of the variants were unstable and produced normal colonies after repeated mass-hyphal subculture on CM. Six phenotypically stable mutants with fawn conidia were derived from strain 649.

No benomyl-resistant mutants of strains 5 and 10 were recovered. This was surprising because they occur in natural populations (see above; Bollen \& Scholten, 1971; Jarvis \& Hargreaves, 1973; Miller \& Fletcher, 1974) and as spontaneous mutants in the laboratory (Polach \& Molin, 1975). Thus, at least some strains of $B$. cinerea must carry appropriate genetic material that is amenable to mutation.

All attempts to isolate auxotrophic mutants of strains 465, 558 and 649 were unsuccessful, and only morphological variants were recovered. Most of the variants were phenotypically unstable. A few of the unstable cultures appeared at first to be nutritional mutants in that they did not grow on unsupplemented media. On further analysis, however, it was found that hyphal subcultures varied considerably: some grew on MM and others were unable to grow on any supplemented media. This result suggests that the medium might be inadequate for selecting auxotrophic mutants. Accordingly, conidial suspensions were exposed to u.v.irradiation and incubated on dishes of MS supplemented with $0.5 \%$ (w/v) Casamino acids, yeast extract and peptone, $0.2 \%(\mathrm{w} / \mathrm{v})$ nucleic acids and $0.1 \%(\mathrm{w} / \mathrm{v})$ caffeine. The additional nutrients were used to provide a very rich medium so that variants with complex nutritional requirements might have a better chance of survival. Caffeine was added because it is known to inhibit DNA repair and, consequently, it can enhance the effects of u.v.irradiation (Hannan \& Nasim, 1977).

No auxotrophic mutants of strain 558 were isolated from the enriched caffeine medium, and most of the morphological variants were unstable. Many of the variants were phenotypically identical, forming colonies with a pronounced red pigment in the mycelium. Similar variants were not obtained by incubating untreated conidia on the caffeine medium, or by incubating u.v.-irradiated conidia on medium without caffeine.

The inability to isolate nutritional and resistance mutants, and the phenotypic instability of most morphological variants, raises fundamental questions regarding the genetic constitution of wild isolates of $B$. cinerea. The results of the mutagenic studies can be accounted for by assuming that isolates are either heterokaryotic or diploid. If they are heterokaryotic, the phenotypic instability of morphological variants could be due to the reassortment of genetically different nuclei during hyphal growth, the phenotype depending on the kinds and proportions of nuclei present; in a balanced lethal system, only heterokaryons would be viable, and pure-breeding homokaryotic progeny would not be recovered. A balanced lethal system would make the isolation of recessive gene mutants very unlikely: a mutation in only one of the nuclear components of a heterokaryon would be masked by the normal allele in the other component (which must be present to retain viability). If wild strains are diploid, the phenotypic instability of induced morphological variants could be due to segregations of haploid, diploid and aneuploid nuclei in the hyphae; stable, recessive gene mutants would be difficult to obtain since the effects of a mutation on one chromosome would be masked by the normal allele on the homologous chromosome. There is evidence that both heterokaryosis and diploidy can contribute to natural variations among populations of fungi (Caten \& Jinks, 1966; Caten \& Day, 1977).

To assess whether the wild strains might be heterokaryotic, asexual progeny from individual cultures on MM and CM were compared for significant differences in morphology and viability. To assess whether they might be diploid, asexual progeny were taken from cultures on PFA-supplemented media; PFA can induce haploidization of diploid fungi (Lhoas, 1961). 

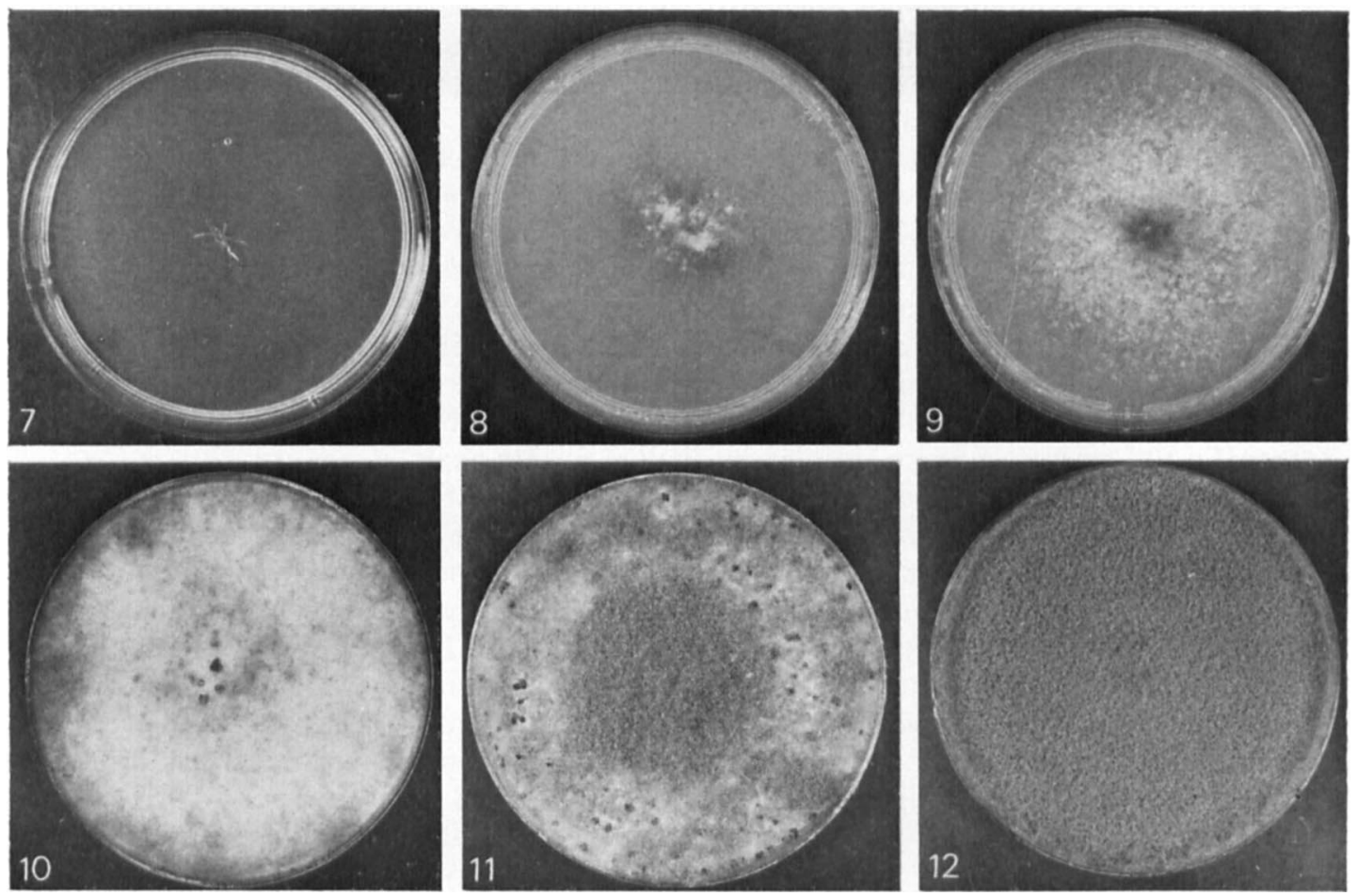

Figs 7 to 12. Variations in growth rate, conidiation and sclerotia production among asexual progeny of $B$. cinerea strain 9 . Each colony was derived from a single macroconidium, incubated on $\mathrm{MM}$ for $14 \mathrm{~d}$ at $25^{\circ} \mathrm{C}$. Only representative colonies are shown: macroconidial progeny exhibited a continuous distribution of phenotypes, from complete inviability to slight growth (Fig. 7 ) to rapid growth and dense conidiation (Fig. 12). Petri dishes are $85 \mathrm{~mm}$ diameter.

\section{Selection experiments}

Six of the wild strains were analysed for variations among macroconidial progeny. The progeny of strains $282,465,558,649$ and 5 differed only slightly in morphology and rate of growth (for example, progeny of strains 465,5 and 558 had growth rates of $11 \cdot 0 \pm 0.05$, $21.6 \pm 0.2$ and $24.7 \pm 0.2 \mathrm{~mm} \mathrm{~d}^{-1}$ at $25^{\circ} \mathrm{C}$, respectively). The conidial progeny of strain 9 , however, differed substantially with respect to general morphology, conidiation and rate of growth (Figs 7 to 12), and included isolates that were inviable (i.e. conidia produced only short germ tubes). Hyphal subcultures of strain 9 also differed substantially, although the phenotypic variations were less extreme than those illustrated in Figs 7 to 12; most of the hyphal tip isolates were inviable, and small groups of hyphae were usually required to establish colonies.

Strains 465, 649, 5 and 9 were grown on PFA media. They all formed colonies with abnormal morphology on CM containing more than $100 \mu \mathrm{g} \mathrm{PFA} \mathrm{m}^{-1}$ (Fig. 14). With few exceptions, the hyphal progeny incubated on dishes of MM, CM, X and Y media were phenotypically wild type. The exceptional progeny produced sectors with unusual conidiation, pigmentation or mycelia, and repeated subculture of the sectored progeny eventually resulted in colonies that differed from the parental strains. For example, the low-conidiation and high-conidiation derivatives of strain 9 (Figs 17,18) were selected from a sectored colony (Fig. 15) on CM; the selected cultures differed from the untreated parental strain (Fig. 13) that was maintained by mass-hyphal subculture.

Conidial variants of strain 9 were selected directly from colonies on CM containing $40 \mu \mathrm{g}$ PFA ml ${ }^{-1}$ : colonies on this medium produced few conidiophores, which were distributed 

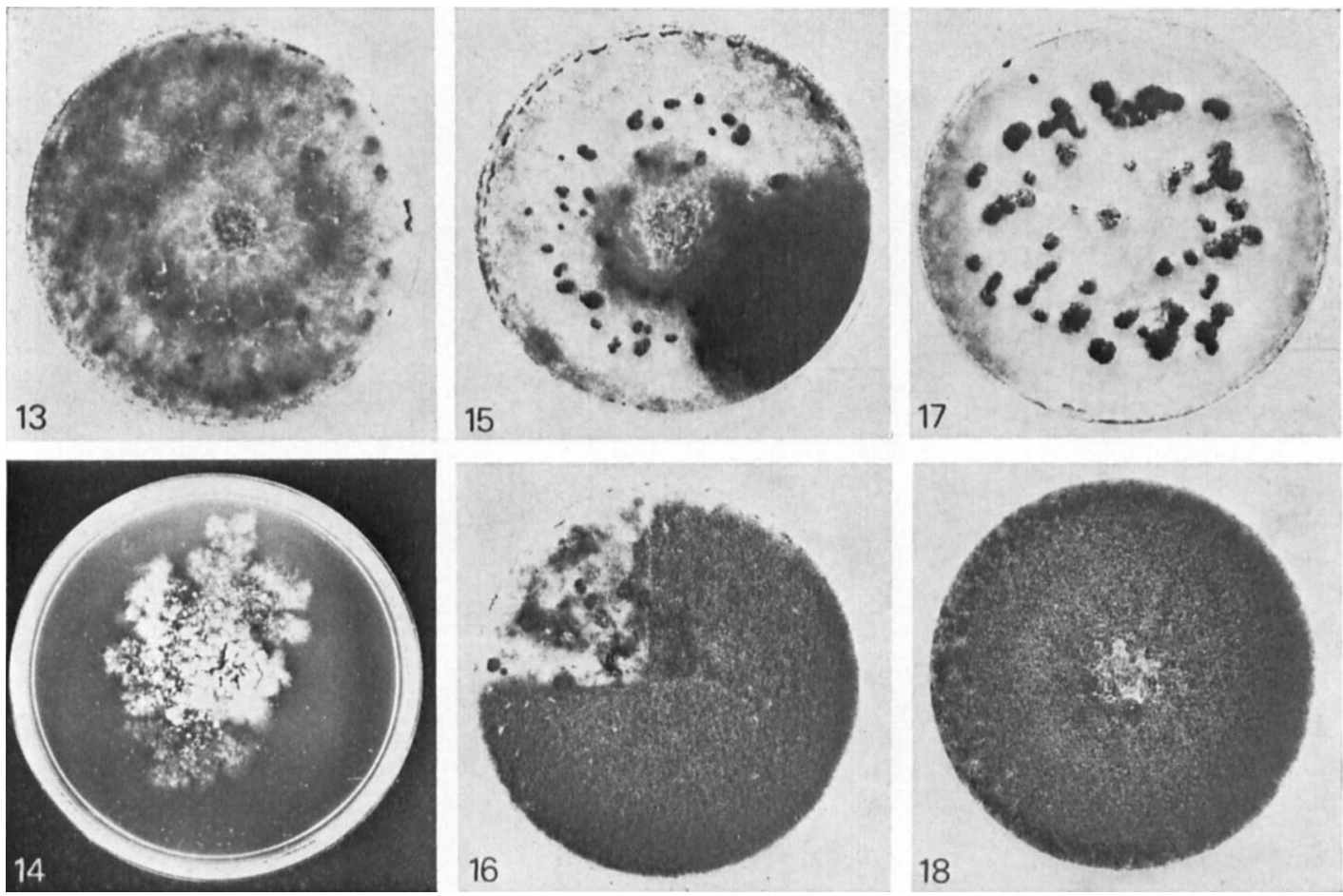

Figs 13 to 18 . Cultures of $B$. cinerea strain 9 before and after selection on medium supplemented with $p$-fluorophenylalanine (PFA). Petri dishes are $85 \mathrm{~mm}$ diameter.

Fig. 13. Typical mass-hyphal subculture on medium $X$, incubated for $21 \mathrm{~d}$ at $25^{\circ} \mathrm{C}$.

Fig. 14. Mass-hyphal subculture on CM containing $160 \mu \mathrm{g} \mathrm{PFA} \mathrm{ml}{ }^{-1}$, incubated for $30 \mathrm{~d}$ at $25^{\circ} \mathrm{C}$. There are very few conidia and no sclerotia.

Figs 15, 16. Progeny derived from hyphae taken from a culture on PFA-supplemented medium. The hyphal inocula were incubated on medium $X$ for $21 \mathrm{~d}$ at $25^{\circ} \mathrm{C}$, and produced colonies with low-conidiation and high-conidiation sectors. Sclerotia on the high-conidiation sectors are masked by dense conidiophores.

Figs 17, 18. Progeny obtained by selection of hyphae and conidia from the sectored colony in Fig. 15. The low-conidiation variant (Fig. 17) was derived from hyphae, and the high-conidiation variant (Fig. 18) from conidia. Inocula were incubated on medium X for $21 \mathrm{~d}$ at $25^{\circ} \mathrm{C}$.

unevenly as tiny clumps against an aconidial background. The hyphal and conidial inocula from these colonies gave rise immediately to low-conidiation and high-conidiation cultures (as in Figs 17, 18), respectively.

The results of the selection experiments do not point conclusively to heterokaryosis or diploidy as the genetic basis for strain variations and phenotypic instability. If strains are diploid, one might expect them to generate many kinds of variant due to haploidization on PFA media; in practice, however, most of the variants were recovered merely by sampling macroconidial and hyphal progeny from cultures on normal media. The phenotypic diversity of conidial isolates of $B$. cinerea was noted by Paul (1929) and Hansen \& Smith (1932). Single conidial isolates studied by Hansen (1938) frequently fell into three phenotypic classes: mycelial (M), conidial (C) and 'intermediate' (MC). He referred to this as the 'dual phenomenon', and suggested that the three phenotypic classes represented the three genotypic classes of progeny of a heterokaryotic parent. Thus, if the parent mycelium contained two kinds of nuclei, A and B, the conidial progeny would comprise A homokaryons and $\mathrm{B}$ homokaryons (phenotypic classes $\mathrm{M}$ and $\mathrm{C}$ ) and $\mathrm{A}+\mathrm{B}$ heterokaryons (phenotypic class MC). A similar conclusion was reached by Webster et al. (1970) in their studies of 

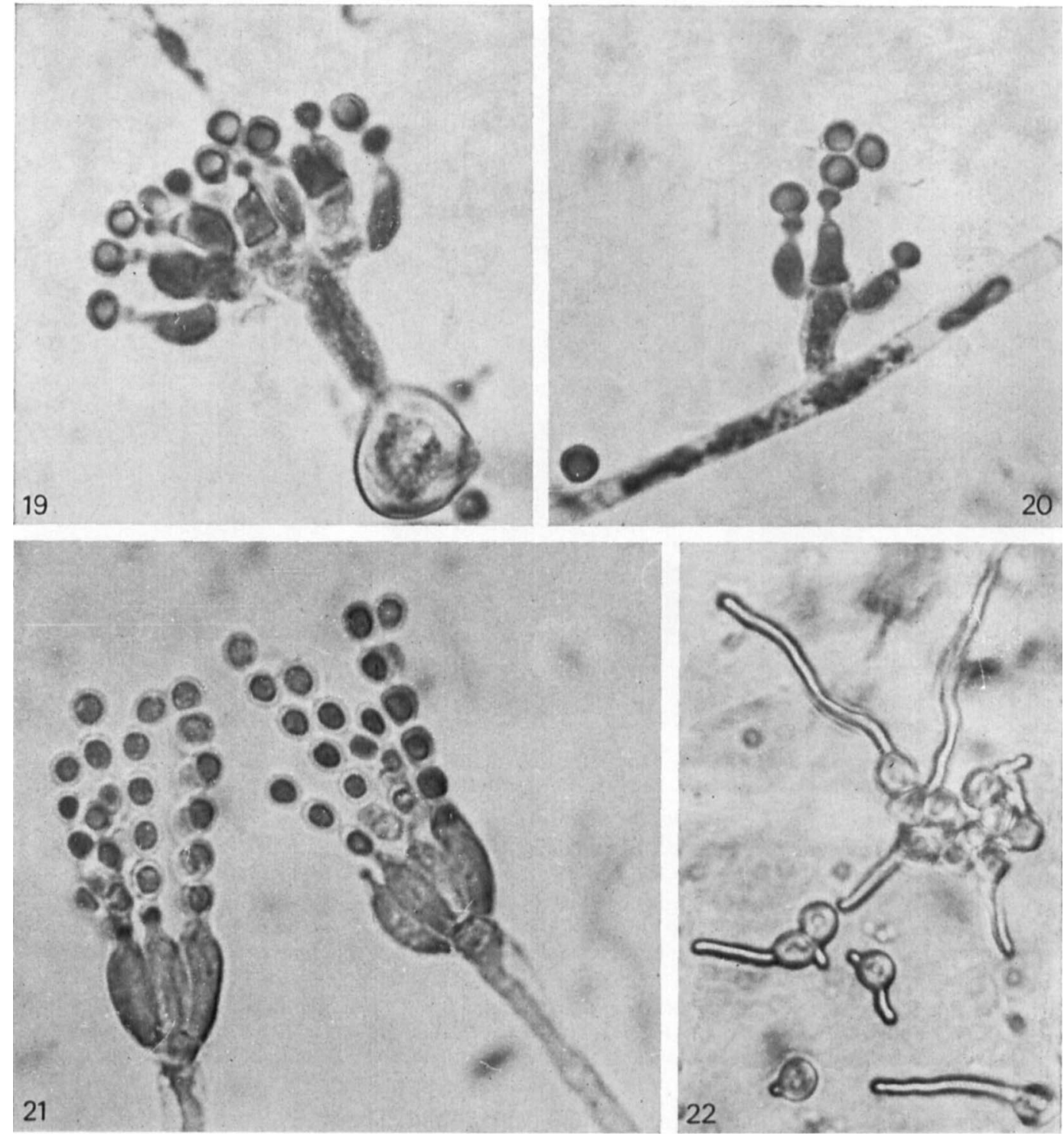

Figs 19 to 22. Photomicrographs of microconidia of B. cinerea strain 649. Microconidia attached to conidiophores are approximately $3 \mu \mathrm{m}$ diameter. Germinated microconidia are swollen, approximately $5 \mu \mathrm{m}$ diameter.

Fig. 19. Microconidiophore arising directly from a germinated macroconidium.

Figs 20, 21. Microconidiophores arising from hyphae, laterally (Fig. 20) and terminally (Fig. 21).

Fig. 22. Microconidia gerninating on CM.

botran-resistant strains of $B$. cinerea selected in the laboratory; they implied that the different levels of resistance shown by conidial progeny were due to segregation of mutant (resistant) and normal (sensitive) nuclei during spore formation by a heterokaryotic parent.

Hansen's explanation can account for some of the variations among asexual progeny described here; in the case of strain 9, the 'inviable' isolates could represent the homokaryotic classes of progeny from a heterokaryon in which the A and B nuclei carry different, complementary lethal genes. However, there is an alternative explanation of the "dual phenomenon': it could represent the selection of different cytoplasmic entities (such as virus-like 
particles, or mitochondria) from a heteroplasmic parent. It is hoped that the heterokaryon versus heteroplasmon question will be resolved by applying the tests suggested by Caten \& Jinks (1966), although progress in this respect will require further studies of the $B$. cinerea strains to clarify such issues as their sexual and heterokaryon compatibility.

The inconclusive data from the analysis of macroconidial progeny focussed attention on the microconidia as a possible source of uninucleate material. A supply of uninucleate spores is more suitable for determining whether homokaryotic progeny are genetically and phenotypically different.

\section{Analysis of microconidia}

The development of microconidiophores was observed on microscope slides coated with water agar, inoculated with macroconidia and incubated at $22{ }^{\circ} \mathrm{C}$ in chambers maintained at 70 to $100 \%$ relative humidity. High humidity ( 95 to $100 \%$ ) was best for the production of microconidia. Microconidiophores developed aerially, on the surface of the agar, and within the medium: they arose from short germ tubes (Fig. 19), from short lateral hyphal branches (Fig. 20) and at the ends of long hyphae (Fig. 21). Mature microconidia were spherical, about $3 \mu \mathrm{m}$ diameter and uninucleate. These studies of microconidia confirm the observations of Brierley (1918).

Microconidial suspensions were incubated at $22{ }^{\circ} \mathrm{C}$ on dishes of $\mathrm{CM}, \mathrm{CMS}$ and water agar. Of the eight strains analysed, only 649 produced microconidia that germinated (Fig. 22), but when further samples of strain 649 microconidia were incubated to derive cultures for analysis, the conidia failed to germinate. Experiments are in progress to determine the precise conditions needed to provide a consistent supply of viable microconidia. The microconidia will be used to derive asexual progeny for comparative studies and as a potential source of induced mutants (it could be significant that the only spore colour mutants were obtained from the only strain, 649, whose microconidia have germinated on agar media).

\section{Crossing experiments}

Attempts to derive sexual progeny by applying conidia to the surface of sclerotia were not successful: no apothecia developed after 3 months. Also, no apothecia were formed in mixed cultures on MM, PDA or sterilized soil after 6 months incubation. This failure to obtain ascospore progeny could be due to the crude experimental method, so it might be necessary to utilize the elaborate system of Groves \& Loveland (1953). It is possible, however, that the strains used here are not sexually compatible; that is, they carry the same mating type allele(s). Such genetic barriers to outcrossing are widespread in fungal populations. In extreme cases, such as Phytophthora infestans, compatible strains with different mating types occur in very few geographical areas (Gallegly \& Niederhauser, 1959). In view of the rarity of the perfect state of $B$. cinerea in nature, it is conceivable that compatible strains of this organism co-exist in few populations. To assess whether this is so, more wild strains of $B$. cinerea will be screened for sexual compatibility. The frequencies and locations of compatible strains should provide a measure of the importance of sexual reproduction as a mechanism for releasing new genetic variants into natural populations.

I am indebted to Hâf Evans, Roy Farrow, Cathie Foot and, especially, to Stephen Oldfield for their technical assistance. I thank the Plant Pathology division of the National Vegetable Research Station, Wellesbourne, for their cooperation in obtaining wild isolates, and the Commonwealth Mycological Institute for identifying the isolates. The photomicrographs were supplied by Stephen Oldfield. 


\section{REFERENCES}

Bollen, G. J. \& Scholten, G. (1971). Acquired resistance to benomyl and some other systemic fungicides in a strain of Botrytis cinerea in cyclamen. Netherlands.Journal of Plant Pathology 77, 83-90.

BrIERLEY, W. B. (1918). The microconidia of Botrytis cinerea. Kew Bulletin of Miscellaneous Information 4, 129-146.

BuRnetT, J. H. (1975). Mycogenetics. London: John Wiley.

Caten, C. E. \& Day, A. W. (1977). Diploidy in pathogenic fungi. Annual Review of Phytopathology 16, 295-318.

CaTEN, C. E. \& Jinks, J. L. (1966). Heterokaryosis: its significance in wild homothallic Ascomycetes and Fungi Imperfecti. Transactions of the British Mycological Society 49, 81-93.

DASSENoY, B. \& MeYer, J. A. (1973). Mutagenic effect of benomyl in Fusarium oxysporum. Mutation Research 21, 119-120.

Davis, R. H. \& DE Serres, F. J. (1970). Genetic and microbiological research techniques for Neurospora crassa. Methods in Enzymology 17, 80-143.

DAY, P. R. (1974). Genetics of Host-Parasite Interaction. San Francisco: W. H. Freeman.

DEKKer, J. (1977). In Systemic Fungicides, 2nd edn, pp. 176-197. Edited by R. W. Marsh. London: Longman.

Drayton, F. L. (1932). The sexual function of the microconidia in certain Discomycetes. Mycologia 24, 345-348.

Gallegly, M. E. \& Niederhauser, J. S. (1959). In Plant Pathology: Problems and Progress 19081958, pp. 168-182. Edited by C. S. Holton. Madison: University of Wisconsin Press.

Greenwood, M. S. \& Berlyn, G. P. (1968). Feulgen cytophotometry of pine nuclei: effects of fixation, role of formalin. Stain Technology 43, 111-117.

Grindle, M. (1973). Sterol mutants of Neurospora crassa: their isolation, growth characteristics and resistance to polyene antibiotics. Molecular and General Genetics 120, 283-290.

GRINDLE, M. (1974). The efficacy of various mutagens and polyene antibiotics for the induction and isolation of sterol mutants of Neurospora crassa. Molecular and General Genetics 130, 81-90.

GrindLe, M. \& Farrow, R. (1978). Sterol content and enzyme defects of nystatin-resistant mutants of Neurospora crassa. Molecular and General Genetics 165, 305-308.

Groves, J. W. \& Drayton, F. L. (1939). The perfect stage of Botrytis cinerea. Mycologia 31, 485-489.

Groves, J. W. \& Loveland, C. A. (1953). The connection between Botryotinia fuckeliana and Botrytis cinerea. Mycologia 45, 415-425.

Hannan, M. A. \& Nasim, A. (1977). Caffeine enhancement of radiation killing in different strains of Saccharomyces cerevisiae. Molecular and General Genetics 158, 111-116.

Hansen, H. N. (1938). The dual phenomenon in imperfect fungi. Mycologia 30, 442-455.

HANSEN, H. N. \& SMITH, R. E. (1932). The mechanism of variation in imperfect fungi: Botrytis cinerea. Phytopathology 22, 953-964.
Hastie, A. C. (1970). Benlate-induced instability of Aspergillus diploids. Nature, London 226, 771.

Hennebert, G. L. (1973). Botrytis and Botrytis-like genera. Persoonia 7, 183-204.

Huebschman, C. (1952). A method for varying the average number of nuclei in the conidia of Neurospora crassa. Mycologia 44, 599-604.

JARVIS, W. R. \& Hargreaves, A. J. (1973). Tolerance to benomyl in Botrytis cinerea and Penicillium corymbiferum. Plant Pathology 22, 139-141.

LAST, F. T. \& Hamley, R. E. (1956). A local lesion technique for measuring the infectivity of the conidia of Botrytis fabae. Annals of Applied Biology 44, 410-418.

LHoAs, P. (1961). Mitotic haploidization by treatment of Aspergillus niger diploids with parafluorophenylalanine. Nature, London 190, 744.

MAJerfeld, I. H. \& Roper, J. A. (1978). The effects of coumarin on the frequency of deletions in a duplication strain of Aspergillus nidulans. Molecular and General Genetics 159, 203-206.

Miller, M. W. \& Fletcher, J. T. (1974). Benomyl tolerance in Botrytis cinerea isolates from glasshouse crops. Transactions of the British Mycological Society 62, 99-103.

Paul, W. R. C. (1929). A comparative morphological and physiological study of a number of strains of Botrytis cinerea Pers. with special reference to their virulence. Transactions of the British Mycological Society 14, 118-135.

Peacock, H. A. (1966). Elementary Microtechniques, 3rd edn. London: Edward Arnold.

Polach, F. J. \& ABawi, G. S. (1975). The occurrence and biology of Botryotinia fuckeliana on beans in New York. Phytopathology 65, 657-660.

Polach, F. J. \& Molin, W. T. (1975). Benzimidazole-resistant mutant derived from a single ascospore culture of Botryotinia fuckeliana. Phytopathology 65, 902-904.

Pontecorvo, G., Roper, J. A., Hemmons, L. M., Macdonald, K. D. \& Bufton, A. W. J. (1953). The genetics of Aspergillus nidulans. Advances in Genetics 5, 141-238.

VOGEL, H. J. (1964). Distribution of lysine pathways among fungi: evolutionary implications. American Naturalist 98, 435-446.

WeAst, R. C. (1967). Handbook of Chemistry and Physics, 48th edn, pE36. Cleveland, Ohio: The Chemical Rubber Co.

Webster, J. (1970). Introduction to Fungi. Cambridge: Cambridge University Press.

Webster, R. K. (1974). Recent advances in the genetics of plant pathogenic fungi. Annual Review of Phytopathology 12, 331-353.

Webster, R. K., Ogawa, J. M. \& Bose, E. (1970). Tolerance of Botrytis cinerea to 2,6-dichloro-4nitroalanine. Phytopathology 60, 1489-1492.

WestergaARD, M. \& Mitchell, H. K. (1947). Neurospora V. A synthetic medium favouring sexual reproduction. American Journal of Botany 34, 573-577. 